\title{
Fatores de Risco para Câncer de Mama em Juiz de Fora (MG): um estudo caso-controle*
}

\author{
Risk factors for breast cancer in Juiz de Fora (MG): a case-control study
}

\author{
Carlos Eduardo Paiva, Bianca Sakamoto Ribeiro, ${ }^{2}$ Agildo Alvarenga Godinho, ${ }^{3}$ Ricardo de Souza Portes Meirelles, \\ Eduardo Vignoli Guzella da Silva, ${ }^{1}$ Guilherme D'Addazio Marques'e Olamir Rossini Júnior ${ }^{5}$
}

\begin{abstract}
Resumo
0 principal fator de risco (FR) para a ocorrência do câncer de mama (CM ) éa idade avançada, porém fatores hormonais endógenos e exógenos, hereditariedade, fatores dietéticos e sóciodemográficos se interagem na etiologia do CM. N osso objetivo foi analisar os fatores de risco para câncer de mama em mulheres de nossa região. Foram entrevistados 80 casos e 76 controles, pareados por idade, consecutivos, de setembro de 2000 a maio de 2001. 0 s dados obtidos foram analisados pelo teste do qui-quadrado e posteriormente pelo modelo de regressão logística. M orar em zona rural foi a variável de risco mais significativa $(p=0,0016)$ e ter tido quatro ou mais filhos mostrou-se como um fator de proteção $(p=0,0094)$. Concluímos que entre as mulheres de nossa região, o fato de morar em zona rural está associado a maior risco de desenvolvimento de CM. Ter tido quatro ou mais filhos, por outro lado, é um fator de proteção.
\end{abstract}

Palavras-chave: neoplasias mamárias; fatores de risco; estudos de casos e controles.

\section{Abstract}

The main risk factor (RF) for the occurence of breast cancer (BC) is old age, although endogenous and exogenous hormones factors, heredity, diet and sociodemographic factors are interrelated in the aetiology of $B C$. O ur aim was to analyse the risk factors for $B C$ in women of our region. Eighty consecutive cases and 76 age matched controls were interviewed, from September 2000 until M ay 2001. The collected data was analysed by chi-squared test and than by the logistic regression model. Living at the countryside was the most significant risk variable $(p=0,0016)$ and having had four or more children seemed to be a protection factor $(p=0,0094)$. We conclude that for women of our region, the fact to live in countryside is associated with a greater risk for developing BC. H aving had four or more children, on the other side, is a protection factor.

Key words breast neoplasms, risk factors, case control studies.

\footnotetext{
*Este trabalho foi comunicado no 120 congresso brasileiro da SBOC.

${ }^{1}$ Acadêmicos da Faculdade de M edicina da Universidade Federal de Juiz de Fora (UFJF) e estagiários do Instituto O ncológico. Enviar correspondência para C.E.P. Rua Paulo Japiassu Coelho, 212 apto 304; 36033-310 Juiz de Fora, M G - Brasil. E-mail: caredupai@bol.com.br

${ }^{2}$ Enfermeira residente em Saúde do Adulto pelo Hospital Universitário da UFJF.

${ }^{3}$ Professor adjunto do departamento de cirurgia da Faculdade de M edicina da UFJF e médico do Instituto O ncológico.

${ }^{4}$ Acadêmico da Faculdade de M edicina da UFJF.

${ }^{5} \mathrm{M}$ édico chefe do serviço de radioterapia do Instituto O ncológico.

Recebido em setembro 2001.
} 


\section{INTRODUÇÃO}

O câncer de mama (CM) é a malignidade mais comum em mulheres no mundo, com um milhão de casos novos a cada ano. ${ }^{1} \mathrm{~N}$ o Brasil, o CM é a primeira causa de morte por câncer entre as mulheres, exceto na região $\mathrm{N}$ orte, onde prevalece 0 câncer do colo de útero. ${ }^{2,3} \mathrm{O} \mathrm{s}$ estados mais desenvolvidos e industrializados, como São Paulo, Rio Grande do Sul e Rio de Janeiro, apresentam a maior incidência da doença. ${ }^{2}$

A etiologia do $\mathrm{CM}$ envolve uma interação de diversos fatores de risco (FR), o que dificulta um estudo mais adequado, pela dificuldade em se isolar um único fator e calcular sua verdadeira contribuição. ${ }^{4,5}$ Além dos fatores endógenos da idade, história familiar e reprodutiva e doença benigna prévia, muitos fatores exógenos estão sendo caracterizados como de risco. D entre estes estão incluídos fatores relacionados à dieta, ${ }^{6}$ exposição à radiação ionizante, ${ }^{1,2,6} 0$ possível o consumo moderado de álcool $\left.\right|^{1,2}$ e exposição a inseticidas organoclorados ${ }^{7-10} M$ utações nos genes BRCA-1 e BRCA-2 são os fatores de risco genéticos mais pesquisados. ${ }^{2} \mathrm{O} C \mathrm{CM}$ ocorre mais em caucasianas e em mulheres de classes sociais mais altas. ${ }^{11}$ Alguns fatores são possivelmente de proteção contra o aparecimento de CM , como alta paridade ${ }^{12-14}$ e lactação. 15,16

0 estudo objetivou determinar quais fatores de risco contribuíram para a ocorrência de CM em mulheres de nossa região geográfica. $\mathrm{N}$ os deteremos aos fatores relacionados à idade, menarca, menopausa, paridade, amamentação, história familiar de CM, idade da primeira gravidez completa, história de abortos e local de moradia (zona rural ou urbana).

\section{MATERIAL E MÉTODOS}

Foram avaliados 80 casos prevalentes de $C M$, internados no Instituto O ncológico de Juiz de Fora (MG) ou em controle ambulatorial na mesma instituição, no período de setembro de 2000 a maio de 2001, desde que fossem do sexo feminino, usuários do Sistema Ú nico de Saúde (SU S) e tivessem diagnóstico histopatológico de câncer de mama. D e forma consecutiva, as pacientes internadas nas enfermarias do hospital e em controle no ambulatório freqüentado pelos estagiários do hospital foram incluídas no estudo. D uas pacientes não responderam 0 questionário devido à gravidade de seus quadros clínicos e outra se recusou. D ois homens portadores de câncer de mama foram excluídos do trabalho. Algumas mulheres não conseguiram responder a todos os itens do questionário, por não se lembrarem de alguns dados retrospectivos. Todas as 80 pacientes analisadas eram usuárias do SU S e concordaram em participar da pesquisa após serem informadas da natureza do estudo. 0 questionário foi aplicado por quatro estagiários de medicina do hospital prétreinados para tal tarefa. 0 mesmo constava de perguntas sobre idade ao diagnóstico, menarca, menopausa, paridade, idade da primeira gravidez completa, amamentação, história familiar para CM, história de qualquer tipo de aborto e local de moradia (urbana ou rural).

0 grupo controle constituiu-se de 76 mulheres, pareadas por idade em relação aos casos e tiveram como critérios de partici pação serem usuárias do SU S e não possuírem história de qualquer tumor em mama. As mulheres do grupo controle foram entrevistadas principalmente em um hospital geral, não relacionado à oncologia e que tem uma área de abrangência semelhante à da instituição onde foram entrevistadas as mulheres do grupo-caso.

Estabelecemos como critério para número de filhos amamentados o período mínimo de seis meses para cada filho. $\mathrm{N}$ ão classificamos os abortos quanto a serem induzidos ou espontâneos. Assim como Brady-West e Graham, ${ }^{6}$ consideramos menarca precoce quando ocorrida com onze anos ou menos e primeira gestação completa tardia quando com trinta anos ou mais. Menopausa tardia foi considerada quando ocorrida com quarenta e cinco anos ou mais, da mesma maneira como foi considerado por $\mathrm{G}$ omes et al ${ }^{11}$

A análise estatística das variáveis estudadas foi feita empregando-se o teste do quiquadrado. Algumas variáveis foram analisadas posteriormente pelo modelo de regressão 
logística. Para os cálculos realizados empregamos o software Statistical Package for Social Science (SPSS for Windows 8.0). Consideramos um nível de significância estatística de 0,05.

\section{RESULTADOS}

A idade média ao diagnóstico foi de 54,5 anos, com um mínimo de 33 anos e máximo de 81 anos. A Tabela 1 resume os dados com relação à idade ao diagnóstico e nos mostra que a faixa etária dos 41 aos 60 anos foi a mais acometida por CM, representando $65 \%(50 / 80)$ do total de mulheres. A Tabela 2 mostra a análise univariada das características de 80 mulheres com CM e seus 76 respectivos controles. Com relação à raça, 45\% (36/80) das mulheres do grupo-caso eram brancas enão houve qualquer tendência a ser este um fator de risco $(p=0,898)$. 0 fato de morar em zona rural foi significativamente mais comum entre as mulheres do grupo-caso $(p=0,013)$. D o total de casos de CM , 27,5\% (22/80) deles se deram em mulheres em pré-menopausa. A idade média da menopausa natural foi de 44,7 anos. Sete mulheres menopausadas não souberam informar a data da última menstruação. A idade da menarca variou entre os casos dos 9 aos 20 anos. $N$ ão houve diferença estatística significativa com relação à menopausa com 45 anos ou mais e menarca com 11 anos ou menos ( $p=0,640$ e $p=0,480$, respectivamente).

Com relação à paridade, as mulheres nulíparas estavam mais presentes no grupo de casos e o fato de terem tido quatro ou mais gestações completas tendeu a ser um fator de proteção, ambos sem diferença significativa $(p=0,222$ e $p=0,083$, respectivamente). A primeira gestação completa após os 30 anos não se mostrou como um FR estatisticamente significativo $(p=0,629)$. Com relação à amamentação, história de abortos e história familiar para $C M$, não houve diferença estatisticamente significativa entre os grupos. Apenas duas mulheres $(2 / 80)$ relataram doença prévia benigna em mama. Analisamos através do modelo de regressão logística todas as variáveis com $p \ll 0,5$ e o resultado é mostrado na tabela
3. Constatamos que o fato de morar em zona rural foi o FR mais significativo $(p=0,0016)$ e ter tido quatro ou mais filhos foi um fator protetor também significativo $(p=0,0094)$.

Tabela 1. Idade ao diagnóstico de 80 mulheres do grupo-caso

\begin{tabular}{c|r|c}
\hline Idade ao diagnóstico & \multicolumn{1}{|c|}{$\mathrm{N}$} & Percentual \\
\hline 31 até 40 anos & $10 / 80$ & $12,50 \%$ \\
\hline 41 até 50 anos & $21 / 80$ & $26,25 \%$ \\
\hline 51 até 60 anos & $29 / 80$ & $36,25 \%$ \\
\hline 61 até 70 anos & $9 / 80$ & $11,25 \%$ \\
\hline 71 até 80 anos & $10 / 80$ & $12,50 \%$ \\
\hline 81 até 90 anos & $1 / 80$ & $1,25 \%$ \\
\hline
\end{tabular}

\section{DISCUSSÃO}

Ainda não foi possível definir lesões verdadeiramente precursoras do $\mathrm{CM}$, ao contrário do que ocorreu com o câncer do colo de útero, vulva e endométrio. ${ }^{2} \mathrm{O}$ estudo ideal dos fatores de risco para CM é difícil, pois estes estão inter-relacionados e 0 isolamento de um único fator para calcular sua real contribuição pode ser impossível. ${ }^{5}$ Para cada estudo mostrando um FR significativo, há outro com resultado diferente. ${ }^{5} \mathrm{~N}$ enhum $\mathrm{FR}$ isolado é tão importante quanto a idade para 0 desenvolvimento do CM.1,5 Alguns autores referem uma curva de idade com dois picos de incidência (um por volta dos 55 anos e outro aos 75 anos). N ossos resultados (Tabela 1) nos levam a concorda com Azzena et al ${ }^{17}$ no que tange à tendência desta curva ser monofásica, com maior incidência por volta dos 50 anos.

U ma atividade cíclica ovariana longa, com uma menarca precoce e/ou menopausa tardia, estão associados a um alto risco de desenvolvimento de CM. ${ }^{17} \mathrm{D}$ ecarli et al ${ }^{13}$ estimaram um decréscimo de $4,7 \%$ no risco de desenvolvimento de CM para cada ano de menarca que se atrasa. Um período fértil de 30 anos ou mais foi relatado como um fator que aumenta em duas vezes a chance de CM $.17 \mathrm{G}$ omes et $\mathrm{a}^{11}$ evidenciaram que ciclos menstruais irregulares são fatores protetores para CM. Em nosso estudo não encontramos diferença significativa referente à menarca com 11 anos ou menos e também da menopausa com 45 anos ou mais, após comparação dos casos em relação aos 
controles. Porém muitas mulheres tiveram dificuldade em se lembrar destas datas, fato sabidamente inerente a este tipo de delineamento estatístico para dados retrospectivos.

Tabela 2. Análise univariada de algumas características das pacientes dos grupos caso e controle

\begin{tabular}{|c|c|c|c|c|c|}
\hline \multirow{2}{*}{ Características } & \multicolumn{2}{|c|}{ Casos } & \multicolumn{2}{|c|}{ Controles } & \multirow{2}{*}{$p$} \\
\hline & $\mathrm{N}$ & $\%$ & $\mathrm{~N}$ & $\%$ & \\
\hline Menarca $<$ ou $=11$ anos & 17 & 22,4 & 12 & 16,4 & 0,480 \\
\hline Menopausa $>$ ou $=45$ anos & 36 & 69,2 & 34 & 75,5 & 0,640 \\
\hline \multicolumn{6}{|l|}{ PARIDADE } \\
\hline Nuliparidade & 15 & 18,7 & 8 & 10,5 & 0,222 \\
\hline 4 ou + gestações completas & 29 & 36,2 & 39 & 51,3 & 0,083 \\
\hline \multicolumn{6}{|c|}{ IDADE EM QUE TEVE O $1^{\circ} \mathrm{FILHO}$} \\
\hline 20 anos ou menos & 25 & 33,3 & 33 & 43,4 & 0,268 \\
\hline 30 anos ou mais & 8 & 10,0 & 5 & 6,6 & 0,629 \\
\hline História de abortos & 16 & 20,0 & 09 & 11,4 & 0,191 \\
\hline \multicolumn{6}{|l|}{ LACTAÇÃO } \\
\hline Nenhum filho & 35 & 43,7 & 35 & 46,0 & 0,898 \\
\hline 5 ou + filhos & 13 & 16,2 & 16 & 21,0 & 0,572 \\
\hline \multicolumn{6}{|l|}{ HISTÓRIA FAMILIAR PARA CM } \\
\hline Em parentes de $1^{\circ}$ grau & 10 & 12,5 & 10 & 13,1 & 0,907 \\
\hline Em parentes de $1^{\circ}$ e $2^{\circ}$ graus & 21 & 26,2 & 17 & 22,3 & 0,705 \\
\hline Morar em zona rural & 14 & 17,5 & 03 & 3,9 & $0,013^{*}$ \\
\hline Raça branca & 36 & 45,0 & 34 & 44,7 & 0,898 \\
\hline
\end{tabular}

Tabela 3. Modelo final da análise por regressão logística das características relacionadas ao $\mathrm{CM}$

\begin{tabular}{l|c}
\hline \multicolumn{1}{c|}{ Características } & $\mathrm{p}$ \\
\hline Menarca com 11 anos ou menos & 0,4440 \\
\hline Nuliparidade & 0,4731 \\
\hline Primeiro filho com 20 anos ou menos & 0,8329 \\
\hline Paridade de 4 ou + filhos & $0,0094^{*}$ \\
\hline História de abortos & 0,1978 \\
\hline Morar em zona rural & $0,0016^{*}$ \\
\hline *Estatisticamente significante para $\mathrm{p}<0,05$ & \\
\hline
\end{tabular}

0 risco de desenvolvimento de $\mathrm{CM}$ em mulheres que tiveram seu primeiro filho após os 30 anos é aproximadamente o dobro do risco das mulheres que 0 fazem antes dos 20 anos. ${ }^{1}$ Segundo Lambe et al, ${ }^{14}$ cada aumento de cinco anos na idade da primeira gravidez sobe o risco de CM em 13\%. Vários estudos concluíram ser a primeira gravidez tardia um fator de risco significativo para CM..$^{12,13,18-20}$ Kalache et al, ${ }^{21}$ em estudo conduzido nas cidades de Fortaleza e Recife, relatou um aumento significativo na ocorrência de CM em mulheres com última gravidez completa tardia, fato também evidenciado previamente por K vale e $\mathrm{H}$ euch. ${ }^{22} \mathrm{~N}$ ossos resultados mostraram não haver significância estatística com relação à idade da primeira gravidez tardia.

Com relação à paridade, um estudo já citado estimou uma diminuição de $10 \%$ no risco de desenvolvimento de CM para cada gestação completa adicional. ${ }^{14} \mathrm{D}$ ecarli et al13 analisaram 2588 casos e concluíram que três ou mais gestações completas é um fator protetor independente para o CM , fato não evidenciado por outro trabalho italiano, que analisou apenas mulheres dos 25 aos 54 anos. ${ }^{12}$ Em nosso estudo, o fato de ter tido quatro ou maisfilhosfoi um fator protetor independente para o desenvolvimento de $\mathrm{CM}$.

A história familiar para CM é um fator de risco bem reconhecido, porém apenas 10 $\%$ das mulheres diagnosticadas com CM têm uma história familiar positiva. M utações dos genes BRCA-1 e BRCA-2 são responsáveis por grande número de casos de $C M$ familiares. ${ }^{1}$ Gomes et al ${ }^{11}$ concluíram que história de $\mathrm{CM}$ em parentes de primeiro grau é um fator de risco independente para a ocorrência de CM . 0 risco de CM é duas vezes maior quando há história familiar para CM em mãe antes dos 40 anos ou irmã, e continua elevado mesmo em mulheres cujo câncer em mãe foi diagnosticado após os 70 anos de idade. ${ }^{23} \mathrm{Em}$ nosso estudo, a história familiar para CM, em parentes de primeiro grau ou em parentes de primeiro e segundo graus, não obteve diferença estatisticamente significante entre os grupos caso e controle.

U ma meta-análise publicada recentemente concluiu que o fator amamentação é protetor para o desenvolvimento do $C M$, porém é de pequena magnitude quando comparado aos FR já estabelecidos. ${ }^{16} \mathrm{O}$ efeito protetor de um longo período de amamentação parece ser mais forte, ou restrito, às mulheres em pré menopausa. ${ }^{15} \mathrm{H}$ ardy et al ${ }^{24}$ concluíram que mulheres que não amamentavam possuíam um risco maior para o desenvolvimento de CM. N ossos resultados, com relação à amamentação, não são condizentes com a literatura atual; porém o critério de amamentação utilizado restringiu-se às mulheres que amamentaram por mais de seis meses e isto provavelmente influenciou 0 resultado.

Com relação à história de abortos, Pike 
et al ${ }^{25}$ concluíram que 0 aborto espontâneo ou induzido no primeiro trimestre da gestação é um FR para CM. Gomes et $\mathrm{al}^{11}$ pesquisaram história de aborto, induzido ou espontâneo, e encontraram um fator protetor à análise univariada que desapareceu após regressão logística. U ma meta-análise conduzida por Brind et al ${ }^{26}$ concluiu que 0 aborto induzido é um FR independente para o desenvolvimento de CM . N ossos resultados não mostraram significância estatística com relação à história de abortos.

A literatura atual nos mostra que o CM é mais comum nos países desenvolvidos e em famílias de classes sociais mais altas. ${ }^{1,11,17}$ Vários estudos mostraram que a incidência de CM é maior em populações urbanas. ${ }^{7,10,27}$ U m estudo norte-americano conduzido na Carolina do $\mathrm{N}$ orte concluiu que morar ou trabalhar na zona rural está associado a um menor risco de desenvolvimento de câncer de mama. ${ }^{7}$ Este mesmo estudo mostrou que dentre um subgrupo de mulheres que trabalhavam na zona rural, aquelas que relataram estar no campo durante ou logo após as aplicações de pesticidas e também aquelas que não utilizaram equipamentos de proteção tiveram um maior risco de desenvolvimento de câncer de mama. ${ }^{7}$ Os inseticidas organoclorados foram largamente utilizados no Brasil, principalmente na década de 70 , na agricultura e também para controle de vetores de doenças. ${ }^{8}$ A tuam como estrogênios fracos e têm como característica principal um al to efeito residual, podendo inclusive permanecer no solo por dezenas deanos, como é o caso do DDT [1,1,1-tricloro-2,2-bis(pclorofenil)etano]. ${ }^{28}$ Olaya-C ontreras et al, ${ }^{9}$ estudando mulheres colombianas, constataram que níveis séricos elevados de DDE [1,1dicloro-2,2-bis(p-clorofenil)etano], principal metabólito do DDT, estão positivamente associados com risco de CM. Este mesmo autor, entretanto, afirma que a relação entre organoclorados e CM é ainda indefinida e enfatiza a necessidade de maiores pesquisas sobre o impacto dos organoclorados na saúde da mulher latino-americana. U m estudo casocontrole recente, ${ }^{8}$ conduzido na cidade do Rio de Janeiro, pesquisou níveis séricos de DDE história de exposição a pesticidas, não estabelecendo qualquer relação causal entre organoclorados e CM. Em nosso estudo o fato de morar em zona rural foi fortemente significativo para o desenvolvimento de CM e isto pode ser campo para novas pesquisas, inclusive com relação à exposição a inseticidas do grupo químico dos clorados e outros FR por nós não pesquisados. Entretanto, nossos resultados podem apenas estar mostrando que o Instituto O ncológico tem uma penetrância maior na zona rural da região em comparação à instituição em que foram entrevistadas as mulheres do grupo-controle, apesar de ambas as instituições serem consideradas de referência para tratamento de pacientes da região.

Apesar do nosso questionário ter sido aplicado diretamente às pacientes de ambos os grupos, há uma chance inerente em qualquer estudo de caso-controle, de erro por parte dos entrevistados, devido à dificuldade de se lembrarem de dados retrospectivos. Nosso estudo pode ter tido este erro, principal mente em relação à idade da menarca e menopausa. Achamos importante enfatizar nossa pequena casuística e a necessidade de estudos maiores e mais detalhados com relação à história de abortos e atividades rurais.

\section{CONCLUSÃO}

Concluímos que em mulheres da nossa população, a faixa etária mais acometida por CM está entre 41 e 60 anos e que 0 fato de morarem em zona rural aumenta de maneira independente 0 risco de desenvolver $\mathrm{CM}$. $M$ ulheres com quatro ou mais filhos possuem um fator protetor para CM. Partindo do principio de que os fatores de risco para $\mathrm{CM}$ são difíceis de serem quantificados e geograficamente variáveis, acreditamos que nossos resultados possam ajudar no entendimento e direcionamento de esforços para uma melhor prevenção do CM em nossa região, com atenção especial às mulheres de zona rural. 


\section{REFERÊNCIAS BIBLIOGRÁFICAS}

1. M cpherson K, Steel CM, D ixon JM. Breast cancer: epidemiology, risk factors, and genetics. BMJ 2000;321:624-8.

2. Hegg R. Câncer de mama. RBM 2000;57(5):463-74.

3. Kligerman J. Estimativas sobre a incidência e mortalidade por câncer no Brasil, 2000. Rev Bras C ancerol 2000;46(2):135-6.

4. M aguireA, $K$ allacheA. Risk factorsfor breast cancer in Brazil [letter]. Int J Epidemiol 1996;25(2):455-6.

5. GomesALRR, Guimarães M D C, G omesCC, Camargos AF. Risk factorsfor breast cancer in Brazil [letter]. Int J Epidemiol 1996;25(2):456-7.

6. Brady-WestD C, G raham SA. Prevalence of risk factors in breast cancer patients at the university hospital of the West Indies. West Indian $M$ ed J 2000;49(2):161-3.

7. Duell EJ, M illikan RC, SavitzD A, N ewman B, Smith JC, Schell M J, et al. A population-based case control study of farming and breast cancer in $\mathrm{N}$ orth Carolina. Epidemiology 2000;11(5):523-31.

8. M endonça $\mathrm{GA}$, Eluf $\mathrm{N}$ eto J, Andrada-Serpa MJ, C armo PA, Barreto H H, Inomata O N, et al. $O$ rganochlorines and breast cancer: a casecontrol study in Brazil. Int J Cancer 1999;83(5):596-600.

9. Olaya-ContrerasP, Rodriguez-Villamil J, PossoValencia HJ, Cortez JE. O rganochlorineexposure and breast cancer risk in Colombian women. C ad Saúde Pública 1998;14(3):12532.

10. Wesseling C, Antich D , H ogstedt C, Rodriguez AC, Ahlbom A. G eographical differences of cancer incidence in Costa Rica in relation to environmental and occupational pesticideexposure. Int J Epidemiol 1999;28(3):365-74.

11. G omes AL, Guimarães MD, G omes CC, Chaves IG, Gobbi H , CamargosAF. A casecontrol study of risk factors for breast cancer in Brazil, 1978-1987. Int J Epidemiol 1995;24(2):292-9.

12. Robertson C, Primic-Zakelj M , BoyleP, H sieh CC. Effect of parity and age at delivery on breast cancer risk in Slovenian women aged 25-54 years. Int J C ancer 1997;73(1):1-9.

13. Decarli A, LaVecchiaC, N egri E, Franceschi S. Ageat any birth and breast cancer in Italy. Int J Cancer 1996;67(2):187-9.
14. Lambe M, H sieh CC, Chan H W, Ekbom A, TrichopoulosD , Adami H O . Parity, ageat first and last birth, and risk of breast cancer: a population-based study in Sweden. Breast C ancer Res Treat 1996;38(3):305-11.

15. Lipworth L, Bailey LR, Trichopoulos D. H istory of breast-feeding in relation to breast cancer risk: a review of the epidemiologic literature. J N atl C ancer Inst 2000;92(4):302-12.

16. Bernier M 0 , Plu-Bureau G , Bossard N , Ayzac $L, T$ halabard $J C$. Breastfeeding and risk of breast cancer: a metaanalysis of published studies. H um Reprod U pdate 2000;6(4):374-86.

17. Azzena A, Zen T, Ferrara A, Brunetti V, Vasile C, M archetti M . Risk factors for breast cancer case-control study results. Eur J G ynaecol O ncol 1994;15(5):386-92.

18. Sancho-Garnier H . Epidemiology of breast cancer. Bull Acad N atl M ed 1998;182(8):162132.

19. Purwanto H, Sadjimin T, D wiprahasto I. Lactation and the risk of breast cancer. $\mathrm{G}$ an To Kagaku Ryoho 2000;27(2):474-81.

20. Ramon JM , EscribajM , CasasI, BenetJ, I glesias C, G avalda L, et al. Ageat first full-term pregnancy, lactation and parity and risk of breast cancer: a case-control study in Spain. Eur J Epidemiol 1996;12(5):449-53.

21. KalacheA, M aguire A, T hompson SG. Ageat last full-term pregnancy and risk of breast cancer. Lancet 1993;341(8836):33-6.

22. Kvale G, H euch I. A prospective study of reproductiverisk factorsand breast cancer. II. Age at first and last birth. Am J Epidemiol 1987;126:842-50.

23. Colditz GA, W illet WC, H unter DJ, Stampfer $M J, M$ anson JE, $H$ ennekens $C H$, et al. Family history, age, and risk of breast cancer. Prospective datafrom the N urses' $\mathrm{H}$ ealth Study. JAM A 1993; 270(13):338-43.

24. $\mathrm{H}$ ardy EE, Pinotti JA, O sis MJ, Fagundes A. Reproductive variables and risk of breast cancer: a case control study carried out in Brazil. Bol O ficina Sanit Panam 1993;115(2):93102.

25. Pike M C, H enderson BE, C asagrande JT, Rosario I, Ray GE. O ral contraceptive use and early abortion as risk factor for breast cancer in young women. BrJ Cancer 1981;43:72-6.

26. Brind J, Chinchilli VM , SeversW B, SummyLong J. Induced abortion as an independent risk factor for breast cancer: a comprehensive review and meta-analysis. J Epidemiol Com- 
munity H ealth 1996;50(5):481-96.

27. Vioque J, Ferrer PJ, Bolumar F. Income, percent of women living in rural areas, parity, and breast cancer mortality in Spain, 1975-1991.
M ed Clin (Barc) 1997;108(2):41-4.

28. Cavero ES, Guerra M S, SilveiraC PD . M anual deinseticidas eacaricidas: aspectostoxicológicos. 1a ed. Pelotas: Aimara; 1976. 